\title{
Symmetry Groups, Similarity Reductions, and Conservation Laws of the Time-Fractional Fujimoto-Watanabe Equation Using Lie Symmetry Analysis Method
}

\author{
Baoyong Guo, ${ }^{1,2}$ Huanhe Dong $\mathbb{D}^{2}, 2$ and Yong Fang $\mathbb{D}^{1,2}$ \\ ${ }^{1}$ College of Electrical Engineering and Automation, Shandong University of Science and Technology, Qingdao 266590, China \\ ${ }^{2}$ College of Mathematics and Systems Science, Shandong University of Science and Technology, Qingdao 266590, China \\ Correspondence should be addressed to Yong Fang; fangyong@sdust.edu.cn
}

Received 25 December 2019; Accepted 28 February 2020; Published 31 March 2020

Academic Editor: Toshikazu Kuniya

Copyright (c) 2020 Baoyong Guo et al. This is an open access article distributed under the Creative Commons Attribution License, which permits unrestricted use, distribution, and reproduction in any medium, provided the original work is properly cited.

In this paper, the time-fractional Fujimoto-Watanabe equation is investigated using the Riemann-Liouville fractional derivative. Symmetry groups and similarity reductions are obtained by virtue of the Lie symmetry analysis approach. Meanwhile, the timefractional Fujimoto-Watanabe equation is transformed into three kinds of reduced equations and the third of which is based on Erdélyi-Kober fractional integro-differential operators. Furthermore, the conservation laws are also acquired by Ibragimov's theory.

\section{Introduction}

Nowadays, nonlinear partial differential equations (NPDEs) have become more and more significant in fluid mechanics, mathematical physics, oceanography, and so on [1-4]. As we know, NPDEs are usually of integer order and researchers have proposed abundant methods to obtain solutions of NPDEs, including inverse scattering transformation [5], Riemann-Hilbert method [6-10], Hirota direct method $[11,12]$, Darboux transformation [13, 14], Bäcklund transformation [15], Frobenius integrable decompositions $[16,17]$, and so on [18-24]. As a generalization, the notions of fractional derivatives are put forward and the classical are Riemann-Liouville and Caputo fractional derivatives. Nonlinear fractional differential equations (NFDEs) are also introduced and have a large number of applications in mathematical physics and automation. The exploration of solutions for NFDEs is a crucial aspect. A variety of methods are presented, for instance, the first integral method [25], functional variable method [26], auxiliary equation method [27], and exponential function method [28].

The Lie symmetry method also provides a way to seek solutions for NPDEs and NFDEs [29-32]. This is a way of using known (old) solutions to find new ones. If we obtain a solution for NPDEs or NFDEs, then by using group transformations, the new solutions can be derived. It means that if a NPDE or NFDE has a solution, then it will actually have infinitely many solutions. This method is so effective. Researchers obtain analytical solutions and conservation laws to equations with the help of this method, for example, seventh-order time-fractional Sawada-Kotera-Ito equation, time-fractional fifth-order modified Sawada-Kotera equation, Burridge-Knopoff equation, and so on [33-37].

The time-fractional Fujimoto-Watanabe equation [38] is

$$
D_{t}^{\gamma} u=u^{3} u_{x x x}+3 u^{2} u_{x} u_{x x}+3 u^{2} u_{x}
$$

where $D_{t}^{\gamma} u$ is the Riemann-Liouville fractional derivative of $u=u(x, t)$ with respect to time variable $t$.

The Fujimoto-Watanabe equation is one important equation and applied in some fields [38]. Its analytical solutions are obtained, and these solutions can reveal many different natural phenomena [39, 40]. For instance, its traveling wave solutions describe the propagation status of water waves in mathematical physics and oceanography. In geography, specialists can predict natural disasters with the 
help of its solutions. In fluid mechanics, researchers acquire its period solutions and study its dynamical behaviors [41].

This paper is organized as follows. In Section 2, we introduce basic concepts and properties about the Riemann-Liouville fractional derivative. In Section 3, symmetry groups are obtained with the help of the Lie symmetry analysis approach. In Section 4, similarity reductions are derived and the time-fractional Fujimoto-Watanabe equation is transformed into three kinds of reduced equations. In Section 5, based on Ibragimov's theory, the conservation laws of the time-fractional Fujimoto-Watanabe equation are constructed. In Section 6, some conclusions are given.

\section{Basic Concept and Properties of the Riemann-Liouville Fractional Derivative}

Definition 1 (Riemann-Liouville fractional derivative) (see [42]). Assuming $f=f(x, t)$ is a real-valued function, where $x$ is the space variable and $t$ is the time variable, then the Riemann-Liouville fractional derivative of $f$ of order $\gamma(\gamma>0)$ is defined as follows:

$$
D_{t}^{\gamma} f(x, t)=\frac{\partial^{\gamma} f}{\partial t^{\gamma}}=\left\{\begin{array}{lr}
\frac{1}{\Gamma(n-\gamma)} \frac{\partial^{n}}{\partial t^{n}} \int_{0}^{t}(t-s)^{n-\gamma-1} f(x, s) \mathrm{d} s, \\
& n-1<\gamma<n, \\
\frac{\partial^{n} f}{\partial t^{n}}, & \gamma=n,
\end{array}\right.
$$

where $\Gamma(x)$ is the gamma function.

The Riemann-Liouville fractional derivative has many properties, for instance,

$$
\begin{aligned}
\text { (a) } D_{t}^{\gamma} t^{\eta} & =\frac{\Gamma(\eta+1) t^{\eta-\gamma}}{\Gamma(\eta-\gamma+1)}, \quad \eta>0, \\
\text { (b) } D_{t}^{\gamma} f[g(t)] & =f_{g}[g(t)] \cdot D_{t}^{\gamma} g(t)=D_{g}^{\gamma} f[g(t)] \cdot\left(g_{t}\right)^{\gamma},
\end{aligned}
$$$$
\text { (c) } D_{t}^{\gamma}[f(t) \cdot g(t)]=D_{t}^{\gamma} f(t) \cdot g(t)+f(t) \cdot D_{t}^{\gamma} g(t) \text {, }
$$

where $f$ and $g$ are real-valued functions, $f_{g}=(\mathrm{d} f / \mathrm{d} g)$, and $g_{t}=(\mathrm{d} g / \mathrm{d} t)$

From Definition 1, we find that the Riemann-Liouville fractional derivative is a generalized form of the ordinary integer-order derivative. Property (b) is the composite rule of the Riemann-Liouville fractional derivative. Property (c) is the Leibniz rule of the Riemann-Liouville fractional derivative.

\section{Symmetry Group of the Time-Fractional Fujimoto-Watanabe Equation}

In this section, we seek symmetry groups for the time fractional Fujimoto-Watanabe equation with the help of the Lie symmetry analysis method.
The general time-fractional differential equation is as follows:

$$
D_{t}^{\gamma} u=L\left(x, t, u, u_{x}, u_{x x}, u_{x x x}, \ldots\right),
$$

where $u=u(x, t)$, the subscripts represent partial derivatives, i.e., $\quad u_{x}=(\partial u / \partial x), u_{x x}=\left(\partial^{2} u / \partial x^{2}\right), u_{x x x}=\left(\partial^{3} u /\right.$ $\left.\partial x^{3}\right), \ldots$, and the corresponding one-parameter transformations are

$$
\begin{aligned}
\bar{x} & =x+\varepsilon \lambda(x, t, u)+O\left(\varepsilon^{2}\right), \\
\bar{t} & =t+\varepsilon \mu(x, t, u)+O\left(\varepsilon^{2}\right), \\
\bar{u} & =u+\varepsilon \varphi(x, t, u)+O\left(\varepsilon^{2}\right), \\
D_{\bar{t}}^{\gamma} \bar{u} & =D_{t}^{\gamma} u+\varepsilon \varphi^{\gamma}(x, t, u)+O\left(\varepsilon^{2}\right), \\
\frac{\partial \bar{u}}{\partial \bar{x}} & =\frac{\partial u}{\partial x}+\varepsilon \varphi^{x}(x, t, u)+O\left(\varepsilon^{2}\right), \\
\frac{\partial^{2} \bar{u}}{\partial \bar{x}^{2}} & =\frac{\partial^{2} u}{\partial x^{2}}+\varepsilon \varphi^{x x}(x, t, u)+O\left(\varepsilon^{2}\right), \\
\frac{\partial^{3} \bar{u}}{\partial \bar{x}^{3}} & =\frac{\partial^{3} u}{\partial x^{3}}+\varepsilon \varphi^{x x x}(x, t, u)+O\left(\varepsilon^{2}\right),
\end{aligned}
$$

where $\varepsilon \ll 1$ is an infinitesimal parameter and $\lambda=\lambda(x, t, u)$, $\mu=\mu(x, t, u)$, and $\varphi=\varphi(x, t, u)$ are real-valued infinitesimal functions with respect to variables $x, t$, and $u$. $\varphi^{\gamma}=\varphi^{\gamma}(x, t, u), \quad \varphi^{x}=\varphi^{x}(x, t, u), \quad \varphi^{x x}=\varphi^{x x}(x, t, u)$, and $\varphi^{x x x}=\varphi^{x x x}(x, t, u)$ are extended infinitesimal functions. These extended infinitesimal functions can be determined using the following expressions:

$$
\begin{aligned}
\varphi^{\gamma}= & D_{t}^{\gamma}(\varphi)+\lambda D_{t}^{\gamma}\left(u_{x}\right)-D_{t}^{\gamma}\left(\lambda u_{x}\right)+D_{t}^{\gamma}\left(D_{t}(\mu) u\right) \\
& -D_{t}^{\gamma+1}(\mu u)+\mu D_{t}^{\gamma+1}(u), \\
\varphi^{x}= & D_{x}(\varphi)-u_{x} D_{x}(\lambda)-u_{t} D_{x}(\mu), \\
\varphi^{x x}= & D_{x}\left(\varphi^{x}\right)-u_{x x} D_{x}(\lambda)-u_{x t} D_{x}(\mu), \\
\varphi^{x x x}= & D_{x}\left(\varphi^{x x}\right)-u_{x x x} D_{x}(\lambda)-u_{x x t} D_{x}(\mu),
\end{aligned}
$$

where $D_{t}^{\gamma}$ represents the total Riemann-Liouville fractional derivative with respect to $t, D_{x}$ represents the total derivative with respect to $x$, and $D_{t}$ represents the total derivative with respect to $t$, i.e.,

$$
\begin{aligned}
& D_{x}=\frac{\partial}{\partial x}+u_{x} \frac{\partial}{\partial u}+u_{x x} \frac{\partial}{\partial u_{x}}+u_{x x x} \frac{\partial}{\partial u_{x x}}+\cdots \\
& D_{t}=\frac{\partial}{\partial t}+u_{t} \frac{\partial}{\partial u}+u_{x t} \frac{\partial}{\partial u_{x}}+u_{x x t} \frac{\partial}{\partial u_{x x}}+\cdots
\end{aligned}
$$

In order to obtain the symmetry groups, let the infinitesimal generator be as follows: 


$$
V=\lambda(x, t, u) \frac{\partial}{\partial x}+\mu(x, t, u) \frac{\partial}{\partial t}+\varphi(x, t, u) \frac{\partial}{\partial u},
$$

where $V$ is sometimes called vector field.

The third-order prolongation of $V$ is

$$
\begin{aligned}
p r^{(3)} V= & \lambda \frac{\partial}{\partial x}+\mu \frac{\partial}{\partial t}+\varphi \frac{\partial}{\partial u}+\varphi^{\gamma} \frac{\partial}{\partial u_{t}^{\gamma}}+\varphi^{x} \frac{\partial}{\partial u_{x}} \\
& +\varphi^{x x} \frac{\partial}{\partial u_{x x}}+\varphi^{x x x} \frac{\partial}{\partial u_{x x x}},
\end{aligned}
$$

where $u_{t}^{\gamma}=D_{t}^{\gamma} u$.

Assume

$$
\begin{aligned}
& \Delta\left(x, t, u, u^{(3)}\right) \\
& =D_{t}^{\gamma} u-L\left(x, t, u, u_{x}, u_{x x}, u_{x x x}, \ldots\right) \\
& =D_{t}^{\gamma} u-u^{3} u_{x x x}-3 u^{2} u_{x} u_{x x}-3 u^{2} u_{x} .
\end{aligned}
$$

In order to satisfy the invariance condition of Lie symmetry, $\operatorname{pr}^{(3)} V$ needs to meet the following identity:

$$
\left.\operatorname{pr}^{(3)} V(\Delta)\right|_{\Delta=0}=0 \text {. }
$$

By direct calculation, we obtain

$$
\begin{gathered}
{\left[\phi^{\gamma}-u^{3} \phi^{x x x}-3 u^{2} u_{x} \phi^{x x}-\left(3 u^{2} u_{x x}+3 u^{2}\right) \phi^{x}\right.} \\
\left.-\left(3 u^{2} u_{x x x}+6 u u_{x} u_{x x}+6 u u_{x}\right) \phi\right]\left.\right|_{\Delta=0}=0
\end{gathered}
$$

From equation (7), we have

$$
\begin{aligned}
\varphi^{x}= & \varphi_{x}+\varphi_{u} u_{x}-\lambda_{x} u_{x}-\lambda_{u} u_{x}^{2}-\mu_{x} u_{t}-\mu_{u} u_{x} u_{t}, \\
\varphi^{x x}= & \varphi_{x x}+\left(2 \phi_{x u}-\lambda_{x x}\right) u_{x}+\left(\varphi_{u u}-2 \lambda_{x u}\right) u_{x}^{2}-\lambda_{u u} u_{x}^{3} \\
& +\left(\varphi_{u}-2 \lambda_{x}\right) u_{x x}-3 \lambda_{u} u_{x} u_{x x}-\mu_{x x} u_{t}-2 \mu_{x} u_{x t} \\
& -2 \mu_{x u} u_{x} u_{t}-2 \mu_{u} u_{x} u_{x t}-\mu_{u} u_{x x} u_{t}-\mu_{u u} u^{2} u_{t}, \\
\varphi^{x x x}= & \varphi_{x x x}+\left(3 \varphi_{x x u}-\lambda_{x x x}\right) u_{x}+3\left(\varphi_{x u u}-\lambda_{x x u}\right) u_{x}^{2} \\
& +\left(\varphi_{u u u}-3 \lambda_{x u u}\right) u_{x}^{3}-\lambda_{u u u} u_{x}^{4}+3\left(\varphi_{x u}-\lambda_{x x}\right) u_{x x} \\
& +3\left(\varphi_{u u}-3 \lambda_{x u}\right) u_{x} u_{x x}-6 \lambda_{u u} u_{x}^{2} u_{x x}+\left(\varphi_{u}-3 \lambda_{x}\right) u_{x x x} \\
& -3 \lambda_{u} u_{x x}^{2}-\mu_{x x x} u_{t}-3 \mu_{x x u} u_{x} u_{t}-3 \mu_{x x} u_{x t} \\
& -6 \mu_{x u} u_{x} u_{x t}-3 \mu_{x} u_{x x t}-3 \mu_{x u u} u_{x}^{2} u_{t}-3 \mu_{x u} u_{x x} u_{t} \\
& -3 \mu_{u u} u_{x}^{2} u_{x t}-3 \mu_{u} u_{x x} u_{x t}-3 \mu_{u} u_{x} u_{x x t} \\
& -3 \mu_{u u} u_{x} u_{x x} u_{t}-\mu_{u} u_{x x x} u_{t}-\mu_{u u u} u_{x}^{3} u_{t}-4 \lambda_{u} u_{x} u_{x x x} .
\end{aligned}
$$

Definition 2 (Generalized Leibniz rule of the Riemann-Liouville fractional derivative). Assuming $u=u(t)$ and $v=v(t)$ are real-valued functions, then the generalized Leibniz rule is

$$
D_{t}^{\gamma}[u(t) \cdot v(t)]=\sum_{n=0}^{\infty}\left(\begin{array}{l}
\gamma \\
n
\end{array}\right) D_{t}^{\gamma-n}[u(t)] \cdot D_{t}^{n}[v(t)], \quad \gamma>0,
$$

where

$$
\left(\begin{array}{l}
\gamma \\
n
\end{array}\right)=\frac{(-1)^{n-1} \gamma \Gamma(n-\gamma)}{\Gamma(1-\gamma) \Gamma(n+1)}
$$

Definition 3 (Generalized composite (chain) rule). Assuming $u=u(t)$ and $v=v(t)$ are real-valued functions, then the generalized composite rule is

$$
\frac{\mathrm{d}^{n} u[v(t)]}{\mathrm{d} t^{n}}=\sum_{i=0}^{n} \sum_{j=0}^{i}\left(\begin{array}{c}
i \\
j
\end{array}\right) \frac{1}{i !}[-v(t)]^{j} \frac{\mathrm{d}^{n}}{\mathrm{~d} t^{n}}\left[(v(t))^{i-j}\right] \frac{\mathrm{d}^{i} u(v)}{\mathrm{d} v^{i}}
$$

Because of equation (11), equation (6) can be rewritten as

$$
\begin{aligned}
\varphi^{\gamma}= & D_{t}^{\gamma}(\varphi)-\gamma D_{t}(\mu) \cdot \frac{\partial^{\gamma} u}{\partial t^{\gamma}}-\sum_{n=1}^{\infty}\left(\begin{array}{c}
\gamma \\
n
\end{array}\right) D_{t}^{n}(\lambda) \cdot D_{t}^{\gamma-n}\left(u_{x}\right) \\
& -\sum_{n=1}^{\infty}\left(\begin{array}{c}
\gamma \\
n+1
\end{array}\right) D_{t}^{n+1}(\mu) \cdot D_{t}^{\gamma-n}(u) .
\end{aligned}
$$

According to equations (11) and (13), we obtain

$$
D_{t}^{\gamma}(\varphi)=\frac{\partial^{\gamma} \varphi}{\partial t^{\gamma}}+\varphi_{u} \frac{\partial^{\gamma} u}{\partial t^{\gamma}}-u \frac{\partial^{\gamma} \varphi_{u}}{\partial t^{\gamma}}+\sum_{n=1}^{\infty}\left(\begin{array}{l}
\gamma \\
n
\end{array}\right) \frac{\partial^{\gamma} \varphi_{u}}{\partial t^{\gamma}} \cdot D_{t}^{\gamma-n}(u)+\phi,
$$

where

$$
\begin{gathered}
\phi=\sum_{n=2}^{\infty} \sum_{i=2}^{n} \sum_{j=2}^{i} \sum_{k}^{j-1}\left(\begin{array}{c}
\gamma \\
n
\end{array}\right)\left(\begin{array}{l}
n \\
i
\end{array}\right)\left(\begin{array}{l}
j \\
k
\end{array}\right) \frac{1}{j !} \frac{t^{n-\gamma}}{\Gamma(n+1-\gamma)} \\
(-u)^{k} \frac{\partial^{i} \varphi}{\partial t^{i}}\left(u^{j-k}\right) \frac{\partial^{n-i+j} \varphi}{\partial t^{n-i} \partial u^{j}} .
\end{gathered}
$$

According to equations (3)b and (3)c, equation (14) can be rewritten as

$$
\begin{aligned}
\varphi^{\gamma}= & \frac{\partial^{\gamma} \varphi}{\partial t^{\gamma}}+\left(\varphi_{u}-\gamma D_{t}(\mu)\right) \frac{\partial^{\gamma} u}{\partial t^{\gamma}}-u \frac{\partial^{\gamma} \varphi_{u}}{\partial t^{\gamma}}+\phi \\
& +\sum_{n=1}^{\infty}\left[\left(\begin{array}{c}
\gamma \\
n
\end{array}\right) \frac{\partial^{n} \varphi_{u}}{\partial t^{n}}-\left(\begin{array}{c}
\gamma \\
n+1
\end{array}\right) D_{t}^{n+1}(\mu)\right] D_{t}^{\gamma-n}(u) \\
& -\sum_{n=1}^{\infty}\left(\begin{array}{c}
\gamma \\
n
\end{array}\right) D_{t}^{n}(\lambda) \cdot D_{t}^{\gamma-n}\left(u_{x}\right) .
\end{aligned}
$$

Substituting equations (14) and (21) into equation (13) and equating the coefficients of all powers of partial 
derivatives of $u$ to 0 , we obtain a set of determining equations as follows:

$$
\begin{aligned}
\lambda_{t} & =\lambda_{u}=0, \\
\mu_{x} & =\mu_{u}=0, \\
\varphi_{u u} & =0, \\
\varphi_{u}-2 \lambda_{x} & =0, \\
3 \lambda_{x}-\gamma \mu_{t}-3 \varphi_{u} & =0, \\
\frac{\partial^{\gamma} \varphi}{\partial t^{\gamma}}-u \frac{\partial^{\gamma} \varphi_{u}}{\partial t^{\gamma}}-u^{3} \varphi_{x x x}-3 u^{2} \varphi_{x} & =0, \\
\left(\begin{array}{c}
\gamma \\
n
\end{array}\right) \frac{\partial^{n} \varphi_{u}}{\partial t^{n}}-\left(\begin{array}{c}
\gamma \\
n+1
\end{array}\right) D_{t}^{n+1}(\mu) & =0 .
\end{aligned}
$$

Solving equation (22), we acquire the solution as follows:

$$
\begin{aligned}
& \lambda(x, t, u)=\gamma C_{1} x+C_{2}, \\
& \mu(x, t, u)=-3 C_{1} t, \\
& \varphi(x, t, u)=2 \gamma C_{1} u,
\end{aligned}
$$

where $C_{1}$ and $C_{2}$ are arbitrary constants.

Based on the above results, the infinitesimal generator can be rewritten as

$$
V=\left(\gamma C_{1} x+C_{2}\right) \frac{\partial}{\partial x}-3 C_{1} t \frac{\partial}{\partial t}+2 \gamma C_{1} u \frac{\partial}{\partial u}
$$

If we let

$$
\begin{aligned}
& V_{1}=\gamma x \frac{\partial}{\partial x}-3 t \frac{\partial}{\partial t}+2 \gamma u \frac{\partial}{\partial u}, \\
& V_{2}=\frac{\partial}{\partial x},
\end{aligned}
$$

then $V$ can also be rewritten as

$$
V=C_{1} V_{1}+C_{2} V_{2} .
$$

Introducing Lie bracket operation, i.e., for arbitrary vector fields $A$ and $B,[A, B]=A B-B A$.

From Table 1, we can find $V_{1}$ and $V_{2}$ are closed obviously. Consequently, the symmetry groups of the timefractional Fujimoto-Watanabe equation can be spanned by $\left\{V_{1}, V_{2}\right\}$.

\section{Similarity Reductions for the Time-Fractional Fujimoto-Watanabe Equation}

In this section, we investigate the similarity reductions for the time-fractional Fujimoto-Watanabe equation. Thus, we can obtain reduced equations.

Because the symmetry groups are spanned by $V_{1}$ and $V_{2}$, we need to discuss in two cases:

Case 1: for $V=(\partial / \partial x)$, we need to solve the following system of equations:
TABLE 1: Lie bracket operation of $V_{1}$ and $V_{2}$.

\begin{tabular}{lcc}
\hline$\left[V_{i}, V_{j}\right]$ & $V_{1}$ & $V_{2}$ \\
\hline$V_{1}$ & 0 & $-\gamma V_{2}$ \\
$V_{2}$ & $\gamma V_{2}$ & 0 \\
\hline
\end{tabular}

$$
\frac{\mathrm{d} x}{1}=\frac{\mathrm{d} t}{0}=\frac{\mathrm{d} u}{0} .
$$

From equation (27), we arrive at $t$ and $u$ are similarity variables. We can assume the solution of equation (1) has the form $u=f(t)$.

Substituting $u=f(t)$ into equation (1), we have the following reduced equation:

$$
D_{t}^{\gamma} u=0
$$

Solving equation (28), we obtain the group invariant solutions:

$$
u=u(x, t)=f(t)=l t^{\gamma-1}
$$

where $l$ is an arbitrary constant.

Case $2(\operatorname{method} 1)$ : for $V=\gamma x(\partial / \partial x)-3 t(\partial / \partial t)+$ $2 \gamma u(\partial / \partial u)$, similar to Case 1, we also need to solve the following system of equations:

$$
\frac{\mathrm{d} x}{\gamma x}=\frac{\mathrm{d} t}{-3 t}=\frac{\mathrm{d} u}{2 \gamma u} \text {. }
$$

From equation (30), we arrive at similarity variables as follows:

$$
\begin{gathered}
\eta=t x^{3 / \gamma} \\
f=u x^{-2}
\end{gathered}
$$

We can assume the solution of equation (1) has the form

$$
u=x^{2} f(\eta)=x^{2} f\left(t x^{3 / \gamma}\right) .
$$

Substituting equation (32) into equation (1), we have the following reduced equation:

$$
\begin{aligned}
D_{\eta}^{\gamma} f(\eta)= & 12 f^{4}+6 f^{3}+\left(\frac{27}{\gamma^{3}}+\frac{81}{\gamma^{2}}+\frac{78}{\gamma}\right) \eta f^{3} f^{\prime} \\
& +\left(\frac{81}{\gamma^{3}}+\frac{81}{\gamma^{2}}\right) \eta^{2} f^{3} f^{\prime \prime}+\frac{27}{\gamma^{3}} \eta^{3} f^{3} f^{\prime \prime \prime} \\
& +\left(\frac{81}{\gamma^{3}}+\frac{81}{\gamma^{2}}\right) \eta^{2} f^{2} f^{\prime 2}+\frac{81}{\gamma^{3}} \eta^{3} f^{2} f^{\prime} f^{\prime \prime}+\frac{9}{\gamma} \eta f^{2} f^{\prime},
\end{aligned}
$$

where $\eta=t x^{3 / \gamma}$. 
For Case 2, we have another method to obtain the reduced equation. We need to use Erdélyi-Kober fractional integro-differential operators.

Case 2 (method 2): for $V=\gamma x(\partial / \partial x)-3 t(\partial / \partial t)+$ $2 \gamma u(\partial / \partial u)$, we also have the following similarity variables:

$$
\begin{aligned}
& \eta=x t^{\gamma / 3}, \\
& f=u t^{(2 / 3) \gamma} .
\end{aligned}
$$

Then, the solution of equation (1) has the form

$$
u=t^{-(2 / 3) \gamma} f(\eta)=t^{-(2 / 3) \gamma} f\left(x t^{(\gamma / 3)}\right) .
$$

Assuming $n-1<\gamma<n$ and substituting equation (35) into Definition 1, we have

$$
D_{t}^{\gamma} u(x, t)=\frac{\partial^{\gamma} u}{\partial t \gamma}=\frac{\partial^{n}}{\partial t^{n}}\left[\frac{1}{\Gamma(n-\gamma)} \int_{0}^{t}(t-\xi)^{n-\gamma-1} \xi^{-(2 / 3) \gamma} f\left(x \xi^{\gamma / 3}\right) \mathrm{d} \xi\right](n-1<\gamma<n) .
$$

Introducing variable transformation,

$$
\mathrm{d} \xi=-\frac{t}{\zeta^{2}} \mathrm{~d} \zeta
$$

$$
\zeta=\frac{t}{\xi}
$$

Substituting equations (37) and (38) into equation (36), we derive

Thus,

$$
\begin{aligned}
D_{t}^{\gamma} u(x, t) & =\frac{\partial^{\gamma} u}{\partial t^{\gamma}}=\frac{\partial^{n}}{\partial t^{n}}\left[t^{n-(5 / 3) \gamma} \frac{1}{\Gamma(n-\gamma)} \int_{1}^{\infty}(\zeta-1)^{n-\gamma-1} \zeta^{-(n-(5 / 3) \gamma+1)} f\left(\zeta^{-(\gamma / 3)} \eta\right) \mathrm{d} \zeta\right] \\
& =\frac{\partial^{n}}{\partial t^{n}}\left[t^{n-(5 / 3) \gamma}\left(K_{-(3 / \gamma)}^{1-(2 / 3) \gamma, n-\gamma} f\right)(\eta)\right] \\
& =\frac{\partial^{n-1}}{\partial t^{n-1}}\left[\frac{\partial}{\partial t}\left(t^{n-(5 / 3) \gamma}\left(K_{-(3 / \gamma)}^{1-(2 / 3) \gamma, n-\gamma} f\right)(\eta)\right)\right] \\
& =\frac{\partial^{n-1}}{\partial t^{n-1}}\left[t^{n-(5 / 3) \gamma-1}\left(n-\frac{5}{3} \gamma+\frac{\gamma}{3} \eta \frac{\mathrm{d}}{\mathrm{d} \eta}\right)\left(K_{-(3 / \gamma)}^{1-(2 / 3) \gamma, n-\gamma} f\right)(\eta)\right],
\end{aligned}
$$

where the definition of the Erdélyi-Kober fractional integral operator is as follows:

$$
\left(K_{k}^{i, j} f\right)(\eta)= \begin{cases}\frac{1}{\Gamma(j)} \int_{1}^{\infty}(\xi-1)^{j-1} \xi^{-(i+j)} f\left(\xi^{1 / k} \eta\right) \mathrm{d} \xi, & j>0, \\ f(\eta), & j=0 .\end{cases}
$$

Repeating the same procedure $n-1$ times, we derive

$$
\begin{aligned}
D_{t}^{\gamma} u(x, t) & =\frac{\partial^{\gamma} u}{\partial t \gamma}=\frac{\partial^{n-1}}{\partial t^{n-1}}\left[t^{n-(5 / 3) \gamma-1}\left(n-\frac{5}{3} \gamma+\frac{\gamma}{3} \eta \frac{\mathrm{d}}{\mathrm{d} \eta}\right)\left(K_{-(3 / \gamma)}^{1-(2 / 3) \gamma, n-\gamma} f\right)(\eta)\right] \\
& =\ldots \\
& =t^{-(5 / 3) \gamma}\left[\prod_{l=0}^{n-1}\left(1-\frac{5}{3} \gamma+l+\frac{\gamma}{3} \eta \frac{\mathrm{d}}{\mathrm{d} \eta}\right)\left(K_{-(3 / \gamma)}^{1-(2 / 3) \gamma, n-\gamma} f\right)(\eta)\right] \\
& =t^{-(5 / 3) \gamma}\left(P_{-(3 / \gamma)}^{1-(5 / 3) \gamma, \gamma} f\right)(\eta),
\end{aligned}
$$


where the definition of the Erdelyi-Kober fractional differential operator is as follows:

$$
\begin{aligned}
\left(P_{k}^{i, j} f\right)(\eta) & =\prod_{l=0}^{n-1}\left(i+l-\frac{1}{k} \eta \frac{\mathrm{d}}{\mathrm{d} \eta}\right)\left(K_{k}^{i+j, n-j} f\right)(\eta), \\
n & = \begin{cases}{[j]+1,} & j \notin \mathbb{N}, \\
j, & j \in \mathbb{N} .\end{cases}
\end{aligned}
$$

Consequently, the time-fractional Fujimoto-Watanabe equation is transformed into the following fractional ordinary differential equation:

$$
\left(P_{-(3 / \gamma)}^{1-(5 / 3) \gamma, \gamma} f\right)(\eta)=f^{3} f^{\prime \prime \prime}+3 f^{2} f^{\prime} f^{\prime \prime}+3 f^{2} f^{\prime},
$$

where $\eta=x t^{(\gamma / 3)}$.

\section{Conservation Laws of the Time-Fractional Fujimoto-Watanabe Equation}

Conservation laws have always been one significant aspect of the investigation on NPDEs and NFDEs. In this section, we construct conservation laws for the time-fractional Fujimoto-Watanabe equation. In this process, Ibragimov's theory plays a key role [43].

Assume

$$
v(x, t)=D_{t}^{\gamma} u-u^{3} u_{x x x}-3 u^{2} u_{x} u_{x x}-3 u^{2} u_{x} .
$$

Introduce a new dependent variable $\xi=\xi(x, t)$ and a formal Lagrangian:

$$
\mathscr{F}=\xi v=\xi\left[D_{t}^{\gamma} u-u^{3} u_{x x x}-3 u^{2} u_{x} u_{x x}-3 u^{2} u_{x}\right] .
$$

We can present action integral of equation (45) in the following form:

$$
\int_{0}^{T} \int_{b}^{a} \mathscr{F}\left(x, t, u, \xi, D_{t}^{\gamma} u, u_{x}, u_{x x}, u_{x x x}\right) \mathrm{d} x \mathrm{~d} t
$$

The corresponding adjoint equation of equation (44) is obtained as follows:

$$
\begin{aligned}
v^{*}= & \frac{\delta \mathscr{F}}{\delta u} \\
= & \left(D_{t}^{\gamma}\right)^{*} \xi+6 u u_{x}^{2} \xi_{x}+6 u^{2} u_{x} \xi_{x x}+3 u^{2} \xi_{x} \\
& +6 u^{2} u_{x x} \xi_{x}+u^{3} \xi_{x x x}=0,
\end{aligned}
$$

where

$$
\begin{aligned}
\frac{\delta}{\delta u} & =\frac{\partial}{\partial u}+\left(D_{t}^{\gamma}\right)^{*} \frac{\partial}{\partial u_{t}^{\gamma}}+\sum_{n=1}^{\infty}(-1)^{n} D_{i_{1}}, \ldots, D_{i_{n}} \frac{\partial}{\partial u_{i_{1}, \ldots, i_{n}}} \\
& =\frac{\partial}{\partial u}+\left(D_{t}^{\gamma}\right)^{*} \frac{\partial}{\partial u_{t}^{\gamma}}-D_{x} \frac{\partial}{\partial u_{x}}+D_{x}^{2} \frac{\partial}{\partial u_{x x}}-D_{x}^{3} \frac{\partial}{\partial u_{x x x}}
\end{aligned}
$$
follows:

$\left(D_{t}^{\gamma}\right)^{*}$ is the adjoint operator of $D_{t}^{\gamma}$, and it is defined as

$$
\left(D_{t}^{\gamma}\right)^{*}=L_{T}^{n-\gamma} D_{t}^{n}
$$

where

$$
L_{T}^{n-\gamma} u(x, t)=\frac{(-1)^{n}}{\Gamma(n-\gamma)} \int_{t}^{T} \frac{u(x, \zeta)}{(\xi-t)^{1+\gamma-n}} \mathrm{~d} \zeta, \quad n-1<\gamma<n .
$$

In order to construct conservation laws, we need to find conservation vector $V^{\sharp}=\left(V^{x}, V^{t}\right)$ which satisfies the following conservation law equation:

$$
\left.\left[D_{x}\left(V^{x}\right)+D_{t}\left(V^{t}\right)\right]\right|_{(1)}=0
$$

where $D_{x}$ and $D_{t}$ are the total derivatives with respect to $x$ and $t$.

According to Ibragimov's theory, conservation vectors can be acquired as follows:

$$
\begin{aligned}
V^{t} & =\mu \mathscr{F}+\sum_{l=0}^{n-1}(-1)^{l} D_{t}^{\gamma-1-l}(\mathscr{R}) D_{t}^{l}\left(\frac{\partial \mathscr{F}}{\partial u_{t}^{\gamma}}\right)-(-1)^{n} J\left[\mathscr{R}, D_{t}^{n}\left(\frac{\partial \mathscr{F}}{\partial u_{t}^{\gamma}}\right)\right] \\
V^{x} & =\lambda \mathscr{F}+\mathscr{R}\left[\frac{\partial \mathscr{F}}{\partial u_{x}}-D_{x}\left(\frac{\partial \mathscr{F}}{\partial u_{x x}}\right)+D_{x} D_{x}\left(\frac{\partial \mathscr{F}}{\partial u_{x x x}}\right)\right]+D_{x}(\mathscr{R})\left[\frac{\partial \mathscr{F}}{\partial u_{x x}}-D_{x}\left(\frac{\partial \mathscr{F}}{\partial u_{x x x}}\right)\right]+D_{x} D_{x}(\mathscr{R})\left[\frac{\partial \mathscr{F}}{\partial u_{x x x}}\right],
\end{aligned}
$$

where $n-1<\gamma<n$ and $\mathscr{R}=\varphi-\lambda u_{x}-\mu u_{t}$ s.t. infinitesimal generator $V=\lambda(\partial / \partial x)+\mu(\partial / \partial t)+\varphi(\partial / \partial u)$, and $J$ operator is defined by

$$
J(u, v)=\frac{1}{\Gamma(n-\gamma)} \int_{0}^{t} \int_{t}^{T} \frac{u(x, \zeta) v(x, \lambda)}{(\lambda-\zeta)^{1+\gamma-n}} \mathrm{~d} \lambda \mathrm{d} \zeta
$$


Case 1: infinitesimal generator $V_{1}=\gamma x(\partial / \partial x)-3 t$

$(\partial / \partial t)+2 \gamma u(\partial / \partial u), \quad \lambda=\gamma x, \quad \mu=-3 t, \quad \varphi=2 \gamma u, \quad$ and

$\mathscr{R}_{1}=2 \gamma u-\gamma x u_{x}+3 t u_{t}$. Then, we have

$$
\begin{aligned}
V^{t}= & -3 t \mathscr{F}+\sum_{l=0}^{n-1}(-1)^{l} D_{t}^{\gamma-1-l}\left(\mathscr{R}_{1}\right) D_{t}^{l}\left(\frac{\partial \mathscr{F}}{\partial u_{t}^{\gamma}}\right)-(-1)^{n} J\left[\mathscr{R}_{1}, D_{t}^{n}\left(\frac{\partial \mathscr{F}}{\partial u_{t}^{\gamma}}\right)\right] \\
= & \sum_{l=0}^{n-1}(-1)^{l} D_{t}^{\gamma-1-l}\left(2 \gamma u-\gamma x u_{x}+3 t u_{t}\right) D_{t}^{l}(\xi)-(-1)^{n} J\left[2 \gamma u-\gamma x u_{x}+3 t u_{t}, D_{t}^{n}(\xi)\right], \\
V^{x}= & \gamma x \mathscr{F}+\mathscr{R}_{1}\left[\frac{\partial \mathscr{F}}{\partial u_{x}}-D_{x}\left(\frac{\partial \mathscr{F}}{\partial u_{x x}}\right)+D_{x} D_{x}\left(\frac{\partial \mathscr{F}}{\partial u_{x x x}}\right)\right]+D_{x}\left(\mathscr{R}_{1}\right)\left[\frac{\partial \mathscr{F}}{\partial u_{x x}}-D_{x}\left(\frac{\partial \mathscr{F}}{\partial u_{x x x}}\right)\right]+D_{x} D_{x}\left(\mathscr{R}_{1}\right)\left[\frac{\partial \mathscr{F}}{\partial u_{x x x}}\right] \\
= & \left(2 \gamma u-\gamma x u_{x}+3 t u_{t}\right)\left[-3 u^{2} u_{x x} \xi-3 u^{2} \xi-D_{x}\left(-3 u^{2} u_{x} \xi\right)+D_{x x}\left(-u^{3} \xi\right)\right] \\
& +D_{x}\left(2 \gamma u-\gamma x u_{x}+3 t u_{t}\right)\left[-3 u^{2} u_{x} \xi-D_{x}\left(-u^{3} \xi\right)\right]+D_{x} D_{x}\left(2 \gamma u-\gamma x u_{x}+3 t u_{t}\right)\left[-u^{3} \xi\right] \\
= & -\left(2 \gamma u-\gamma x u_{x}+3 t u_{t}\right)\left(3 u^{2} \xi+3 u^{2} u_{x} \xi_{x}+3 u^{2} u_{x x} \xi+u^{3} \xi_{x x}\right)+\left(\gamma u_{x x}-\gamma x u_{x x}+3 t u_{x t}\right)\left(u^{3} \xi_{x}\right)+\left(\gamma x u_{x x x}-3 t u_{x x t}\right)\left(u^{3} \xi\right) .
\end{aligned}
$$

Case 2: infinitesimal generator $V_{2}=(\partial / \partial x), \lambda=1$, $\mu=0, \varphi=0$, and $\mathscr{R}_{2}=-u_{x}$. Then, we have

$$
\begin{aligned}
V^{t} & =\sum_{l=0}^{n-1}(-1)^{l} D_{t}^{\gamma-1-l}\left(\mathscr{R}_{2}\right) D_{t}^{l}\left(\frac{\partial \mathscr{F}}{\partial u_{t}^{\gamma}}\right)-(-1)^{n} J\left[\mathscr{R}_{2}, D_{t}^{n}\left(\frac{\partial \mathscr{F}}{\partial u_{t}^{\gamma}}\right)\right] \\
& =\sum_{l=0}^{n-1}(-1)^{l+1} D_{t}^{\gamma-1-l}\left(u_{x}\right) D_{t}^{l}(\xi)-(-1)^{n+1} J\left[u_{x}, D_{t}^{n}(\xi)\right] \\
V^{x} & =\mathscr{F}+\mathscr{R}_{2}\left[\frac{\partial \mathscr{F}}{\partial u_{x}}-D_{x}\left(\frac{\partial \mathscr{F}}{\partial u_{x x}}\right)+D_{x} D_{x}\left(\frac{\partial \mathscr{F}}{\partial u_{x x x}}\right)\right]+D_{x}\left(\mathscr{R}_{2}\right)\left[\frac{\partial \mathscr{F}}{\partial u_{x x}}-D_{x}\left(\frac{\partial \mathscr{F}}{\partial u_{x x x}}\right)\right]+D_{x} D_{x}\left(\mathscr{R}_{2}\right)\left[\frac{\partial \mathscr{F}}{\partial u_{x x x}}\right] \\
& =\left(-u_{x}\right)\left[-3 u^{2} u_{x x} \xi-3 u^{2} \xi-D_{x}\left(-3 u^{2} u_{x} \xi\right)+D_{x x}\left(-u^{3} \xi\right)\right]+D_{x}\left(-u_{x}\right)\left[-3 u^{2} u_{x} \xi-D_{x}\left(-u^{3} \xi\right)\right]+D_{x} D_{x}\left(-u_{x}\right)\left[-u^{3} \xi\right] \\
& =\left(u_{x}\right)\left(3 u^{2} \xi+3 u^{2} u_{x} \xi_{x}+3 u^{2} u_{x x} \xi+u^{3} \xi_{x x}\right)-\left(u_{x x}\right)\left(u^{3} \xi_{x}\right)+\left(u_{x x x}\right)\left(u^{3} \xi\right) \\
& =3 u^{2} u_{x} \xi+3 u^{2} u_{x} u_{x x} \xi+3 u^{2} u_{x}^{2} \xi_{x}+u^{3} u_{x} \xi_{x x}-u^{3} u_{x x} \xi_{x}+u^{3} u_{x x x} \xi
\end{aligned}
$$

\section{Conclusions}

In this investigation, we explore the time-fractional Fujimoto-Watanabe equation in the perspective of the Riemann-Liouville derivative. We acquire symmetry groups and similarity reductions by means of the Lie symmetry approach. Meanwhile, three kinds of reduced equations have been obtained. For the second infinitesimal generator, we use two distinct methods to derive two different reduced equations, one of which is based on fractional integral operators and fractional differential operators. At last, with the help of Ibragimov's theory, the conservation laws are constructed. These results reveal this approach is very effective to obtain reduced equations for the fractional differential equations.

\section{Data Availability}

No data were used to support this study.

\section{Conflicts of Interest}

The authors declare that there are no conflicts of interest. 


\section{Acknowledgments}

This work was supported by the National Natural Science Foundation of China (Grant nos. 11975143 and 61602188), Shandong Provincial Natural Science Foundation (Grant no. ZR2019QD018), and Scientific Research Foundation of Shandong University of Science and Technology for Recruited Talents (Grant nos. 2017RCJJ068 and 2017RCJJ069).

\section{References}

[1] B. B. Kadomtsev and V. I. Petviashvili, "On the stability of solitary waves in weakly dispersive media," Soviet Physics-Doklady, vol. 15, pp. 539-541, 1970.

[2] J. Satsuma and M. J. Ablowitz, "Two-dimensional lumps in nonlinear dispersive systems," Journal of Mathematical Physics, vol. 20, no. 7, pp. 1496-1503, 1979.

[3] D. A. Benson, S. W. Wheatcraft, and M. M. Meerschaert, "Application of a fractional advection-dispersion equation," Water Resources Research, vol. 36, no. 6, pp. 1403-1412, 2000.

[4] C. Rotschild, M. Segev, Z. Xu, Y. V. Kartashov, L. Torner, and O. Cohen, "Two-dimensional multipole solitons in nonlocal nonlinear media," Optics Letters, vol. 31, no. 22, pp. 3312$3314,2006$.

[5] M. J. Ablowitz, D. J. Kaup, A. C. Newell, and H. Segur, “The inverse scattering transform-fourier analysis for nonlinear problems," Studies in Applied Mathematics, vol. 53, no. 4, pp. 249-315, 1974.

[6] M. A. Efendiev and W. L. Wendland, "Nonlinear RiemannHilbert problems for multiply connected domains," Nonlinear Analysis: Theory, Methods \& Applications, vol. 27, no. 1, pp. 37-58, 1996.

[7] T. Liu and H. Dong, "The prolongation structure of the modified nonlinear Schrödinger equation and its initialboundary value problem on the half line via the RiemannHilbert approach," Mathematics, vol. 7, no. 2, p. 170, 2019.

[8] W.-Q. Peng, S.-F. Tian, X.-B. Wang, T.-T. Zhang, and Y. Fang, "Riemann-Hilbert method and multi-soliton solutions for three-component coupled nonlinear Schrödinger equations," Journal of Geometry and Physics, vol. 146, Article ID 103508, 2019.

[9] Y. Lin, H. Dong, and Y. Fang, "N-soliton solutions for the NLS-like equation and perturbation theory based on the Riemann-Hilbert problem," Symmetry, vol. 11, no. 6, p. 826, 2019.

[10] Y. Zhang, H.-H. Dong, and D.-S. Wang, "Riemann-Hilbert problems and soliton solutions for a multi-component cubicquintic nonlinear Schrödinger equation," Journal of Geometry and Physics, vol. 149, Article ID 103569, 2020.

[11] R. Hirota, The Direct Method in Soliton Theory, Cambridge University Press, Cambridge, UK, 2004.

[12] B. Y. Guo, H. H. Dong, and Y. Fang, "Lump solutions and interaction solutions for the dimensionally reduced nonlinear evolution equation," Complexity, vol. 2019, Article ID 5765061, 9 pages, 2019.

[13] V. B. Matveev and M. A. Salle, Darboux Transformations and Solitons, Springer-Verlag, Berlin, Germany, 1991.

[14] C. H. Gu and Z. X. Zhou, "On Darboux matrices of Bäcklund transformations for AKNS systems," Letters in Mathematical Physics, vol. 13, pp. 179-187, 1987.
[15] C. Rogers and W. K. Schief, Bäcklund and Darboux Transformations: Geometry and Modern Applications in Soliton Theory, Cambridge University Press, Cambridge, UK, 2002.

[16] W.-X. Ma, H. Wu, and J. He, "Partial differential equations possessing Frobenius integrable decompositions," Physics Letters A, vol. 364, no. 1, pp. 29-32, 2007.

[17] Y. Fang, H. Dong, Y. Hou, and Y. Kong, "Frobenius integrable decompositions of nonlinear evolution equations with modified term," Applied Mathematics and Computation, vol. 226, pp. 435-440, 2014.

[18] E. Fan, "Extended tanh-function method and its applications to nonlinear equations," Physics Letters A, vol. 277, no. 4-5, pp. 212-218, 2000.

[19] Y. Chen and B. Li, "General projective Riccati equation method and exact solutions for generalized KdV-type and $\mathrm{KdV}$-Burgers-type equations with nonlinear terms of any order," Chaos, Solitons \& Fractals, vol. 19, no. 4, pp. 977-984, 2004.

[20] Y. Kong and Y. Fang, "Behavior of the correction equations in Jacobi-Davidson method," Mathematical Problems in Engineering, vol. 2019, Article ID 5169362, 4 pages, 2019.

[21] B. Ren, W.-X. Ma, and J. Yu, "Rational solutions and their interaction solutions of the $(2+1)$-dimensional modified dispersive water wave equation," Computers \& Mathematics with Applications, vol. 77, no. 8, pp. 2086-2095, 2019.

[22] P. J. Olver, Applications of Lie Groups to Differential Equations, Springer-Verlag, Berlin, Germany, 2000.

[23] Y. Tang, M. Yuen, and L. Zhang, "Double wronskian solutions to the $(2+1)$-dimensional Broer-Kaup-Kupershmidt equation," Applied Mathematics Letters, vol. 105, Article ID 106285, 2020.

[24] L. Zhang, Y. Shi, M. Han et al., "Smooth and singular traveling wave solutions for the Serre-Green-Naghdi equations," Discrete \& Continuous Dynamical Systems-S, 2018.

[25] N. A. Kudryashov, "Lax pair and first integrals of the traveling wave reduction for the KdV hierarchy," Applied Mathematics and Computation, vol. 350, pp. 323-330, 2019.

[26] A. D. Polyanin, "Functional separable solutions of nonlinear convection-diffusion equations with variable coefficients," Communications in Nonlinear Science and Numerical Simulation, vol. 73, pp. 379-390, 2019.

[27] S. Guo and Y. Zhou, "Auxiliary equation method for the mKdV equation with variable coefficients," Applied Mathematics and Computation, vol. 217, no. 4, pp. 1476-1483, 2010.

[28] B. Wang and X. Wu, "Exponential collocation methods for conservative or dissipative systems," Journal of Computational and Applied Mathematics, vol. 360, pp. 99-116, 2019.

[29] E. Yaşar and T. Özer, "Invariant solutions and conservation laws to nonconservative FP equation," Computers \& Mathematics with Applications, vol. 59, no. 9, pp. 3203-3210, 2010.

[30] E. Yaşar and T. Özer, "Conservation laws for one-layer shallow water wave systems," Nonlinear Analysis: Real World Applications, vol. 11, no. 2, pp. 838-848, 2010.

[31] S. Sahoo and S. Saha Ray, "The conservation laws with lie symmetry analysis for time fractional integrable coupled KdV-mKdV system," International Journal of Non-linear Mechanics, vol. 98, pp. 114-121, 2018.

[32] M. Guo, H. Y. Dong, J. X. Liu, and H. W. Yang, “The timefractional mZK equation for gravity solitary waves and solutions using sech-tanh and radial basis function method," Nonlinear Analysis: Modelling and Control, vol. 24, pp. 1-19, 2019. 
[33] E. Yaşar, "Variational principles and conservation laws to the Burridge-Knopoff equation," Nonlinear Dynamics, vol. 54, no. 4, pp. 307-312, 2008.

[34] J. Hu, Y. Ye, S. Shen, and J. Zhang, "Lie symmetry analysis of the time fractional KdV-type equation," Applied Mathematics and Computation, vol. 233, pp. 439-444, 2014.

[35] E. Yaşar, Y. Yıldırım, and C. M. Khalique, "Lie symmetry analysis, conservation laws and exact solutions of the seventhorder time fractional Sawada-Kotera-Ito equation," Results in Physics, vol. 6, pp. 322-328, 2016.

[36] E. Yaşar and Y. Yıldırım, "On the Lie symmetry analysis and traveling wave solutions of time fractional fifth-order modified Sawada-Kotera equation," Karaelmas Science and Engineering Journal, vol. 8, pp. 411-416, 2018.

[37] C. Lu, L. Xie, and H. Yang, "Analysis of Lie symmetries with conservation laws and solutions for the generalized $(3+1)$ dimensional time fractional Camassa-Holm-KadomtsevPetviashvili equation," Computers \& Mathematics with Applications, vol. 77, no. 12, pp. 3154-3171, 2019.

[38] C.-s. Liu, "Applications of complete discrimination system for polynomial for classifications of traveling wave solutions to nonlinear differential equations," Computer Physics Communications, vol. 181, no. 2, pp. 317-324, 2010.

[39] L.-J. Shi and Z.-S. Wen, "Bifurcations and dynamics of traveling wave solutions to a Fujimoto-Watanabe equation," Communications in Theoretical Physics, vol. 69, no. 6, p. 631, 2018.

[40] H. Dong, Y. Fang, B. Guo, and Y. Liu, "Lie point symmetry, conservation laws and exact power series solutions to the Fujimoto-Watanabe equation," Quaestiones Mathematicae, pp. 1-17, 2019.

[41] L. J. Shi and Z. S. Wen, "Several types of periodic wave solutions and their relations of a Fujimoto-Watanabe equation," Journal of Applied Analysis and Computation, vol. 9, no. 4, pp. 1193-1203, 2019.

[42] I. Podlubny, Fractional Differential Equations, Academic Press, San Diego, CA, USA, 1999.

[43] N. H. Ibragimov, "A new conservation theorem," Journal of Mathematical Analysis and Applications, vol. 333, no. 1, pp. 311-328, 2007. 